\title{
Optimization of multi-response dynamic systems integrating multiple regression and Taguchi's dynamic signal-to-noise ratio concept
}

\author{
Surajit Pal $^{1 *}$, Susanta Kumar Gauri ${ }^{2}$ \\ ${ }^{1 *}$ SQC \& OR Unit, Indian Statistical Institute, 110, N. M. Road, Chennai 600 029, INDIA \\ ${ }^{2}$ SQC \& OR Unit, Indian Statistical Institute, 203, B.T. Road, Kolkata 700 108, INDIA \\ "Corresponding Author: e-mail: surajitpal@ hotmail.com, Tel + 9444539260
}

\begin{abstract}
The principal difference between a dynamic and a static system is that the former includes a signal factor for expressing the intended output while the later does not. Assuming a linear association exists between the response and signal variables, Taguchi offered a two-stage route for optimizing a dynamic system: maximize the dynamic signal-to noise ratio (DSN) and then, change the gradient to the desired gradient by a suitable modification parameter. Some researchers have indicated limitations to Taguchi's DSN analysis, and advocated alternative approaches for optimization of a dynamic system. However, the Taguchi method as well as these alternative approaches is useful for optimizing a single-response dynamic system only. In realism, the majority of the contemporary manufacturing practices encompass numerous response variables as well as industries demand for developing procedures for optimizing multi-response dynamic system. This paper proposes a novel procedure that integrates multiple regression (MR) technique and Taguchi's DSN concept to optimize the multi-response dynamic system. In this method, appropriate multiple regression equations according to a chosen model for dynamic system are fitted first based on the observed experimental data and then DSN (called MRDSN) for different response variables are computed using the MRbased predicted values. Finally, weighted MRDSN is considered as the objective function for the optimization. The proposed procedure is investigated with respect to three modelling approaches for the dynamic systems. The results of analysis reveal that the proposed procedure with response modelling approach results in the best optimization performance. It also results in better optimization performance than back-propagation neural network-based approach and data mining-based approach reported by the past researchers.
\end{abstract}

Keywords: multiple responses, multiple regression, weighted dynamic signal-to-noise ratio, performance measure modelling, response function modelling, response modelling, optimization

DOI: http://dx.doi.org/10.4314/ijest.v9i1.2

\section{Introduction}

Dynamic systems are those where target values of one or more response variables depend on the setting of the signal factor. Examples of a signal factor may be the steering angle in the steering mechanism of an automobile or the speed control setting of a fan. Product/process design with a dynamic system offers the flexibility needed to satisfy customer requirements and can enhance a manufacturer's competitiveness. Taguchi (1990) first took interest in designing robust dynamic systems. Assuming that a linear relationship exists between the response variable and the signal factor of the system, Taguchi proposed a two-step procedure for optimizing a dynamic system: maximize the dynamic signal-to noise ratio (DSN) and then, adjust the slope to the desired slope by a suitable adjustment factor. A control factor that has a large effect on the slope but no effect on DSN is considered as an adjustment factor. Some successful applications of Taguchi method for optimizing single-response dynamic systems are available in Tzeng and Chiu (2003), Wu et al. (2005) and Tzeng and Jean (2005).

In light of the increasing demands of the customers, the need for dynamic systems is increasing rapidly in industries. In recent time, therefore, many other researchers (Miller and Wu, 1996; Tsui, 1998; Tsui, 1999; Wasserman, 1996; McCaskey and Tsui, 
1997; Joseph and Wu, 2002; Lesperance and Park, 2003; Bae and Tsui, 2006; Dasgupta et al., 2010) have been motivated to study the robust design problem concerning the dynamic systems. These researchers have highlighted the potential problems of Taguchi's DSN analysis and advocated some alternative approaches for optimization of a dynamic system. The alternative approaches studied by Miller and Wu (1996), Tsui (1998), Tsui (1999), McCaskey and Tsui (1997), Joseph and Wu (2002) and Dasgupta et al. (2010) differ from Taguchi's approach mainly with respect to the modelling approaches. In the Taguchi method, DSN, i.e. $10 \log _{10}\left(\beta^{2} / \sigma_{\beta}^{2}\right)$, where $\beta$ is the slope of the ideal relationship and $\sigma_{\beta}^{2}$ is the variance around the slope, is modelled as a function of control factors. On the other hand, in the alternative approaches, $\beta^{2}$ and $\sigma_{\beta}^{2}$ are modelled separately as functions of control factors (known as performance measure modelling), or control and noise factors (known as response function modelling) or the response is directly modelled as a function of control, noise and signal factors (known as response modelling). In the alternative approaches, the factors levels that minimize variance is chosen first and then slope is adjusted to its target slope by using suitable adjustment factor. Wasserman (1996) explained the use of Taguchi's DSN using a regression perspective. Lesperance and Park (2003) proposed the use of a joint generalized model. Bae and Tsui (2006) generalized the response modelling approach. On the other hand, Su et al. (2005) and Jung and Yum (2011) proposed artificial neural network based approaches for optimizing a dynamic system. These alternative approaches can overcome many potential problems of the Taguchi method. However, all these research articles are focused on optimization of a single-response dynamic system.

Product/process design has a tendency of being somewhat multifaceted to satisfy persistently shifting needs of the customer as well as manufacturing know-how. Manifold reactions should essentially be appraised concurrently to resolve complete process/product value (Tong et al., 2008). Industries have, therefore, all the time more highlighted building up measures competent of concurrently optimizing the multi-response dynamic systems in the light of the increasing complexity of modern product design. To deal with the requirements of the contemporary industries, more than a few studies have (Tong et al., 2002; Tong et al., 2004; Hsieh et al., 2005; Wang and Tong, 2005; Wu and Yeh, 2005; Chang, 2006; Wang, 2007; Wu, 2007; Chang, 2008; Tong et al., 2008; Wu, 2009; Zhong and Liu, 2009; Chang and Chen, 2011; Wang et al., 2011; Sharma et al., 2012; Gauri, 2014; Zhang et al., 2014; Wu, 2015; He et al., 2015; Bashiri et al., 2016) have proposed different procedures for optimizing a multi-response dynamic system. Applying the two-step procedure is not feasible for optimizing a multi-response dynamic system because it is almost impossible to find out any control factor that may be considered as an adjustment factor. Therefore, the basic approach adopted by the researchers is to define first an appropriate overall objective function (performance metric) and then to optimize the objective function such that each response variable is as close as possible to its target value with minimum variability around the target value at each signal level.

The available approaches for the optimization of dynamic systems can be broadly classified into three categories: (1) mathematical modelling based approaches (Tong et al., 2002; Hsieh et al., 2005; Wu, 2007; Zhang et al., 2014; Bashiri et al., 2016), (2) multiple attribute decision making (MADM) methods based approaches (Tong et al., 2004; Wang and Tong, 2005; Wang, 2007; Zhong and Liu, 2009; Gauri, 2014; Wu, 2015) and (3) meta-heuristic approaches (Chang, 2006; Chang, 2008; Tong et al., 2008; Chang and Chen, 2011). The implementation of the mathematical modelling based approaches requires that the engineers possess a well-built experience in mathematics or statistics, whereas the MADM method based approaches are easy to implement but the optimal solution derived by these approaches may not guarantee that all response means are near to their relevant goal values at different signal levels. On the other hand, in meta-heuristic based approaches, the actual association amid the response variables and control factors remain unknown, and therefore, the engineers fail to gain knowledge of professional engineering practices for the duration of the optimizing process. Therefore, the process engineers are even now looking for an effortlessly implementable scheme capable of optimizing the manifold responses, certifying that no particular response move away remotely from its goal and unevenness in the region of the goal is the least amount at all signal level along with understanding of the actual relationship between the response variables and control factors.

Pal and Gauri (2010) showed that, in case of a multi-response static problem, multiple regression based weighted signal-to-noise ratio (MRWSN) method can results in an optimal solution which ensure that every quality features (responses) are extremely near to their relevant goal values having sensibly little inconsistency in the region of the goal values. In this technique, suitable polynomial regression expressions for prediction of mean and variance of all response variables are established first based on the experimental data and then the weighted signal-to-noise ratio (WSN) is computed using the predicted mean and variance of the response variables, and optimized. Since the method includes establishment of the regression equations, the process engineers also get clear understanding of the actual relationship between the response variables and control factors during the process of optimization. The purpose of the present investigation is to extend the concept of Pal and Gauri (2010) in solving multi-response dynamic problem so that the gap between the available techniques and the process engineers' requirements in solving multiresponse dynamic problems can be fulfilled.

The research report is ordered as follows. Section 2 outlines succinctly the Taguchi's dynamic system and reports various approaches for its optimization. Section 3 presents the review of the literature on the optimization of multi-response dynamic systems. The proposed method for optimization of a multi-response dynamic system under different approaches for modelling the dynamic system is presented in section 4. In section 5, analysis of one experimental dataset taken from literature and related results are discussed. The paper is concluded in section 6 . 


\section{Taguchi's Dynamic System and Related Works}

Robust design problem of a dynamic system was first addressed by Taguchi (1990). He considered a dynamic system where a single response variable is expected to assume different target values as a result of changes in the levels of a signal factor. A signal factor (e.g. speed controller of a fan) is a factor that is controlled by the user of the product to make use of its intended function.

According to Taguchi (1990), perfect quality is established on the perfect association amid the signal factor as well as the response variable, and quality loss is brought about through deviations from the perfect association. So, noteworthy quality enhancement is deemed to be attained through first describing a system's perfect function and after that, employing designed experiments to seek an optimal design which decreases deviations from this perfect function. Taguchi (1990) supposed that a linear relationship is present amid the response variable $(Y)$ and the signal factor $(M)$ of the system, and thus the perfect function could be articulated thus:

$$
Y=\beta M+\varepsilon
$$

where $\beta$ is the slope or system sensitivity, and $\varepsilon$ denotes random error. Here $\varepsilon$ is assumed to follow a normal distribution with a mean of zero and variance of $\sigma_{\beta}^{2}$. The deviation from the ideal function is represented by the variability of the dynamic system, i.e. $\sigma_{\beta}^{2}$.

For obtaining the robust design of a product/process, Taguchi suggested a special experimental format (i.e. cross-product of inner and outer arrays) and analysis of experimental data. In the inner array control factors are assigned and in the outer array signal and noise factors are assigned. For each combination of control factor levels in the inner array, values of the response variable under all the combinations of signal and noise factor levels in the outer array are measured, and these observations are used to estimate the slope $(\beta)$ and variance $\left(\sigma_{\beta}^{2}\right)$ of the ideal function. Thus, for an experiment involving one $s$-level signal factor $\left(M_{1}, M_{2}, \ldots, M_{s}\right), n$-level noise factor settings $\left(Z_{1}, Z_{2}, \ldots, Z_{n}\right)$ and with $r$ number of repeated observed values, the slope $(\beta)$ and variance $\left(\sigma_{\beta}^{2}\right)$ of a response $(Y)$ in a fixed combination of control factor settings (levels) are estimated as follows:

$$
\begin{gathered}
\beta=\frac{\sum_{k=1}^{s} \sum_{l=1}^{n} \sum_{m=1}^{r}\left(y_{k l m} M_{k}\right)}{\sum_{k=1}^{s} M_{k}^{2}} \\
\sigma_{\beta}^{2}=\frac{1}{s n r-1} \sum_{k=1}^{s} \sum_{l=1}^{n} \sum_{m=1}^{r}\left(y_{k l m}-\beta M_{k}\right)^{2}
\end{gathered}
$$

where $y_{k l m}$ is the observed value of the response variable $(Y)$ in the $m^{\text {th }}$ replication at $k^{\text {th }}$ level of signal factor and $l^{\text {th }}$ level of noise factor setting. The aspiration of robust design is the discovery of the amalgamation of control factor levels such that the consequence of noise factors on the target response of the dynamic system is trivial (Chang, 2008). Taguchi, therefore, recommended to use DSN to judge the system's performance. For a fixed combination of control factor levels, the DSN is obtained as follows:

$$
D S N=10 \log _{10}\left(\frac{\beta^{2}}{\sigma_{\beta}^{2}}\right)
$$

Taguchi (1990) treats the computed DSN values corresponding to different combinations of control factor levels in the inner array as the responses, and proposes the following two-step procedure for identifying the optimal factor settings. In the first step, the settings of the control factors that maximizes DSN is determined and in the second step, the slope $(\beta)$ is shifted to the target slope by changing the level of the adjustment factor (a control factor that has a large effect on $\beta$ but no effect on DSN).

Wasserman (1996) observed that the factor-level combination of a dynamic system using Taguchi's DSN might not be optimal. Lunani et al. (1997) noted that using DSN as a quality performance measure might produce inaccuracies due to biased dispersion effect. Miller and Wu (1996) have highlighted that Taguchi (1990) essentially proposed a performance measure modelling (PMM) approach for dynamic system optimization. In the PMM approach, the performance measures (PMs) are modelled as functions of the control factor effects, and Taguchi (1990) considers the DSN as the PM. Tsui (1998) considers that DSN is a loss measure by which a system's robustness is quantified, and so he has termed Taguchi's approach as the loss model (LM) approach. It is not always possible to identify an ideal signal-response relationship and the assumption of the existence of an adjustment factor also does not hold in many practical situations. Therefore, Miller and $\mathrm{Wu}(1996)$ recommend to consider the intercept, slope ( $\beta$ ) and variability $\left(\sigma_{\beta}^{2}\right)$ as the separate PMs for the purpose of modelling and analysis. They have also proposed a new modelling approach, called response function modelling (RFM), which models the PMs as functions of the effects of both the control and 
noise factors. The advantage of the RFM approach is that it can reveal how a specific control factor interacts with a specific noise factor. Tsui (1998) has investigated optimization of dynamic systems using response model (RM) which directly models the response as a function of the control, noise and signal factors.

Tsui (1999) has derived relationship between the effect estimates of the LM approach and those of the RM approach. Tsui (1998) has compared the performance of LM, RFM and RM approaches. He has found that the LM approach creates unnecessary biases for the factorial effect estimates and may lead to non-optimal solutions and information loss. McCaskey and Tsui (1997) have developed a two-step procedure for optimization of dynamic systems under an additive model. Joseph and Wu (2002) have formulated the robust parameter design of dynamic system as a mathematical programming problem. Lesperance and Park (2003) have suggested to use a joint generalized linear model (GLM) so that model assumptions can be investigated using residual analysis. Su et al. (2005) and Jung and Yum (2011) have applied neural network based approaches to optimize parameter design with dynamic characteristics. Bae and Tsui (2006) have observed that the GLM-RM approach can provide more reliable results. Dasgupta and $\mathrm{Wu}$ (2010) have developed statistical models and performance measures for measurement systems, a kind of dynamic system. All these research articles are focussed on optimization of a single-response dynamic system.

\section{Review of Literature on Optimization of Multi-response Dynamic Systems}

The problem of optimization of multi-response dynamic system has drawn lesser attention of the researchers. Tong et al. (2002) have used dual-response-surface method (RSM) and composite desirability function (CDF) for optimization of a multi-response dynamic system. Tong et al. (2004) utilizes principal component analysis (PCA) to simplify the dynamic multi-response problems and applied TOPSIS (technique for order preference by similarity to ideal solution) (Hwang and Yoon, 1981; Hwang et al., 1993) to derive the overall performance index for optimization. Hsieh et al. (2005) have made use of regression analysis to examine the noteworthy control factors influencing the deviations as well as responsiveness of a dynamic system and then employed CDF for optimization. Wang and Tong (2005) have incorporated the TOPSIS and multiple attribute decision making (MADM) method into the grey relational model (Deng, 1982) for determining the optimal parameter setting. Wu and Yeh (2005) derived total quality loss and minimized it to determine the optimal settings for a multi-response dynamic system. Chang (2006) has proposed an artificial neural network (ANN) approach for modelling the response functions and has applied CDF for optimization of the multiresponse dynamic systems. Wang (2007) has developed a procedure of optimizing multi-response dynamic systems using PCA and multiple criteria evaluation of the grey relation model. Wu (2007) extends the Taguchi's quality loss function for multiple dynamic quality characteristics and builds a polynomial regression model to optimize the parameter design. Tong et al. (2008) optimized a dynamic system using data envelopment analysis (DEA). Chang (2008) has proposed a data mining method comprising of a four-stage system established on ANN, exponential desirability functions and a simulated annealing (SA) procedure to determine the optimization problem of multi-response dynamic systems. Wu (2009) has presented an approach to optimize non-linear multiple dynamic quality characteristics based on double-exponential desirability function. Chang and Chen (2011) have proposed a crossbreed method that contains exponential desirability functions in a neuro-genetic method to optimize dynamic multi-response systems. Wang et. al. (2011) presented an integrated approach that utilizes desirability function, principal component analysis, grey relational analysis and TOPSIS method. Zhang et al. (2014) have proposed a modified particle swarm optimization algorithm for dynamic multi-response optimization based on goal programming approach. Gauri (2014) considered overall utility value as the objective function for optimization of multi-response dynamic systems. Wu (2015) proposed a proportion of quality loss (PQL) model for optimization of a multi-response dynamic system. Bashiri et.al. (2016) proposed a procedure for optimising multi-response dynamic system using multivariate process capability index.

Most of the researchers (Tong et al, 2002; Hsieh et al, 2005; Chang, 2006; Chang, 2008; Wu, 2009; Chang and Chen, 2011) have attempted to optimize multi-response dynamic systems using Derringer and Suich's (1980) composite desirability function (CDF) as a performance metric. The basic advantage of using CDF as performance metric is that it is a simple unit less measure and it has a good foundation in statistical practice. However, one of the serious limitations of CDF is that it does not consider the variability of individual response variables. Moreover, if the requirement restrictions regarding the response variables were not offered, the CDF cannot be computed. On the other hand, grey relational grade, overall utility value, PQL etc. that are used as a performance metric in MADM method based approaches (Tong et al., 2004; Wang and Tong, 2005; Wang, 2007; Zhong and Liu, 2009; Gauri, 2014; $\mathrm{Wu}, 2015$ ) may lead to an optimal solution where one or more individual response move far away from their respective target values.

\section{Proposed Method for optimization of Multi-response Dynamic Systems}

The main idea behind the proposed approach is to compute DSN for different response variables using some predicted values obtained by appropriately fitted multiple regression equations and then to construct an overall performance index based on these computed DSN values. It may be noted that past researchers have advocated three types of modelling approaches for the singleresponse dynamic system, e.g. 1) PMM, 2) RFM and 3) RM. In PMM approach, $\beta$ and $\sigma_{\beta}^{2}$ are modelled as functions of the control factors only. In RFM approach, $\beta$ and $\sigma_{\beta}^{2}$ are modelled as functions of control factors and noise factor settings. On the other hand, in RM approach, the response $Y$ is directly modelled as function of the control, noise and signal factors. Therefore, 
multiple regression equations may be established for prediction of $\beta$ and $\sigma_{\beta}^{2}$ values based on control factor settings or multiple regression equations may be established for prediction of $\beta$ and $\sigma_{\beta}^{2}$ based on control factor settings and noise level settings, or multiple regression equation may be established for prediction of a response $(Y)$ based on control factor settings, noise level settings and signal level. Therefore, for a fixed control factor settings, DSN for a response variable can be obtained by using directly the predicted $\beta$ and $\sigma_{\beta}^{2}$ values or by using $\beta$ and $\sigma_{\beta}^{2}$ that can be computed from predicted response values. Since, in this process, DSN values are computed using multiple regression-based predicted values, it may be called as multiple regressionbased dynamic signal-to-noise ratio (MRDSN). For a multi-response dynamic system, the weighted MRDSN (WMRDSN) can be taken as overall objective function or performance metric for optimization.

\subsection{Establishing multiple regression equations under different modelling approaches}

Suppose that in a multi-response dynamic system, there are $p$ output responses $\left(Y_{1}, Y_{2} \ldots, Y_{p}\right), q$ control factors $\left(X_{1}, X_{2}, \ldots, X_{q}\right)$ each with two/three number of levels, a signal factor with $s$ levels $\left(M_{1}, M_{2}, \ldots, M_{s}\right)$ and $n$ noise factor settings $\left(Z_{1}, Z_{2}, \ldots, Z_{n}\right)$. Also suppose that $t$ trial conditions or experimental runs for the inner array of control factors are conducted each with $r$ replications to obtain the experimental data. The data in different experimental runs can be recorded in the format shown in Table 1. In this table, the observed values in a trial conditions for $i^{\text {th }}(i=1,2,3, \ldots, p)$ response variable in $m^{\text {th }}(m=1,2,3, \ldots, r)$ replication at $k^{\text {th }}(k=1,2,3, \ldots, s)$ level of signal factor and $l^{\text {th }}(l=1,2,3, \ldots, n)$ level of noise factor setting is denoted by $y_{i k l m}$.

Table 1. Data format for a multi-response dynamic system

\begin{tabular}{|c|c|c|c|c|c|c|c|c|c|c|c|c|}
\hline \multirow{3}{*}{$\begin{array}{l}\text { Trial } \\
\text { No. }\end{array}$} & \multirow{2}{*}{\multicolumn{4}{|c|}{ Control factors }} & \multirow{3}{*}{$\begin{array}{l}\text { Noise } \\
\text { factor } \\
\text { settings }\end{array}$} & \multicolumn{7}{|c|}{ Responses } \\
\hline & & & & & & \multicolumn{3}{|c|}{$Y_{1}$} & \multirow{2}{*}{$\begin{array}{l}\cdots \\
\ldots\end{array}$} & \multicolumn{3}{|c|}{$Y_{p}$} \\
\hline & $X_{1}$ & $X_{2}$ & $\ldots$ & $X_{q}$ & & $M_{1}$ & $\cdots$ & $M_{s}$ & & $M_{1}$ & $\ldots$ & $M_{s}$ \\
\hline \multirow{3}{*}{1} & & & & & $Z_{1}$ & $\begin{array}{c}y_{1111} \\
\ldots \\
y_{111 r}\end{array}$ & $\ldots$ & $\begin{array}{c}y_{1 s 11} \\
\ldots \\
y_{1 s 1 r}\end{array}$ & $\ldots$ & $\begin{array}{c}y_{p 111} \\
\ldots \\
y_{p 11 r}\end{array}$ & $\ldots$ & $\begin{array}{c}y_{p s 11} \\
\ldots \\
y_{p s 1 r}\end{array}$ \\
\hline & & & & & $\ldots$ & $\ldots$ & $\ldots$ & $\ldots$ & $\ldots$ & $\ldots$ & $\ldots$ & $\ldots$ \\
\hline & & & & & $Z_{n}$ & $\begin{array}{c}y_{11 n 1} \\
\ldots \\
y_{11 n r}\end{array}$ & $\ldots$ & $\begin{array}{c}y_{1 s n 1} \\
\ldots \\
y_{1 s n r}\end{array}$ & $\ldots$ & $\begin{array}{c}y_{p 1 n 1} \\
\ldots \\
y_{p 1 n r}\end{array}$ & ... & $\begin{array}{c}y_{p s n 1} \\
\ldots \\
y_{p s n r}\end{array}$ \\
\hline$\ldots$ & $\ldots$ & $\ldots$ & $\ldots$ & $\ldots$ & $\ldots$ & $\ldots$ & $\ldots$ & $\ldots$ & $\ldots$ & $\ldots$ & $\ldots$ & $\ldots$ \\
\hline$\ldots$ & $\ldots$ & $\ldots$ & $\ldots$ & $\ldots$ & $\cdots$ & $\ldots$ & $\cdots$ & $\ldots$ & $\cdots$ & $\ldots$ & $\cdots$ & $\cdots$ \\
\hline$t$ & $\ldots$ & $\ldots$ & $\ldots$ & $\ldots$ & $\ldots$ & $\ldots$ & $\ldots$ & $\ldots$ & $\ldots$ & $\ldots$ & $\cdots$ & $\ldots$ \\
\hline
\end{tabular}

\subsubsection{Multiple regression equation for prediction under PMM approach}

In an experimental run, there are $s \times n \times r$ observations for each response variable. Based on these observations, compute the slope $(\beta)$ and variance $\left(\sigma_{\beta}^{2}\right)$ values for each response variable in the run using Eqns. (2) and (3) respectively, and let these values in the run for $p$ response variables $Y_{i}(i=1,2, \ldots, p)$ are denoted as $\beta_{y_{i}}$ and $\sigma_{\beta_{y_{i}}}^{2}$ respectively. Similarly, for all the $t$ experimental runs, compute $\beta_{y_{i}}$ and $\sigma_{\beta_{y_{i}}}^{2}(i=1,2, \ldots, p)$ values. Develop now the following regression model for prediction of $\beta_{y_{i}}$ under an experimental run:

$$
\beta_{y_{i}}=b_{0}+\sum_{u=1}^{p} b_{u} X_{u}+\sum_{u, v} b_{u v} X_{u} X_{v}+\varepsilon
$$


and the following regression model for prediction of $\sigma_{\beta_{y_{i}}}^{2}$ value:

$$
\log _{10}\left(\sigma_{\beta_{y_{i}}}^{2}\right)=c_{0}+\sum_{u=1}^{p} c_{u} X_{u}+\sum_{u, v} c_{u v} X_{u} X_{v}+\varepsilon
$$

where $b_{u}, c_{u}, b_{u v}, \quad c_{u v}(u=1,2, \ldots, q ; v=1,2,3, \ldots, q)$ are regression coefficients, $X_{u}(u=1,2, \ldots, q)$ are controllable variables, $\varepsilon$ is the random error term which is assumed to follow a normal distribution with mean value as zero and a constant variance $\sigma^{2}$. It may be noted that apart from the main factors, the quadratic terms of main factors may be included in the model when there is three or more number of levels in any control factor. The interaction terms of controllable variables may also be included in the model.

It may be noticed that corresponding to $t$ experimental runs there are values of $\beta_{y_{i}}$ and $\sigma_{\beta_{y_{i}}}^{2}$ for each response variable $Y_{i}(i=1,2, \ldots, p)$. Based on these values, the regression coefficients of the regression models for each response variable can easily be obtained by using the least squares method. The choice of carrying out multiple linear regression analysis is obtainable in Microsoft Excel as well as in lots of statistical software packages, e.g. SPSS, MINITAB, STATISTICA, etc. The coefficient of determinations, $R^{2}$ and adjusted $R^{2}$, are taken into account to abandon needless model's expressions and to incorporate merely those expressions which contain a number of contributions on the dependent variable. In addition, scientific matters should be employed to incorporate any expression into the regression model. Going by a rule of thumb, if the value of $R^{2}$ is more than 0.90 and the value of adjusted $R^{2}$ is more than 0.80 , the fitted model is considered adequate.

Problem-solving confirmation for endorsing the regression models ought to be executed. The satisfactoriness of the regression model could be confirmed by carrying out significance tests employing ANOVA as well as F-test. In an effort to identify likely irregularities, a residual analysis from the perspective of a variety of plots, for instance, residuals' normality plot, residual plot against predicted values as well as residual plot against individual regression variable, etc. should be observed. Following the problem-solving confirmation, if no grave infringement of model suppositions is noticed, subsequently, the regression expression could be supposed to be sufficiently robust to forecast the dependent variable. More details about fitting of multiple regression equations and the diagnostic checks are available in Montgomery et al. (2012).

Suppose that finally established (fitted) regression equations for prediction of $\beta_{y_{i}}$ and $\sigma_{\beta_{y_{i}}}^{2}(i=1,2, \ldots, p)$ are as follows:

$$
\begin{aligned}
& \beta_{y_{i}}=\hat{b}_{0}+\sum_{u=1}^{q} \hat{b}_{u} X_{u}+\sum_{u, v} \hat{b}_{u v} X_{u} X_{v} \\
& \log _{10}\left(\sigma_{\beta_{y_{i}}}^{2}\right)=\hat{c}_{0}+\sum_{u=1}^{q} \hat{c}_{u} X_{u}+\sum_{u, v} \hat{c}_{u v} X_{u} X_{v}
\end{aligned}
$$

where $\hat{b}_{u}, \hat{c}_{u}, \hat{b}_{u v}, \hat{c}_{u v}(u=1,2, \ldots, q ; v=1,2,3, \ldots, q)$ are the estimates of the regression coefficients.

\subsubsection{Multiple regression equations for prediction under RFM approach}

In a noise level under an experimental run, there are $s \times r$ observations for each response variable. Based on these observations, slope and variance values can be computed for each response variable in the noise level under the experimental run. Let the slope and variance of $i^{\text {th }}$ response variable $Y_{i}(i=1,2,3, \ldots, p)$ in the $l^{\text {th }}(l=1,2, \ldots, n)$ noise level under the experimental run are denoted as $\beta_{y_{i l}}$ and $\sigma_{\beta_{y_{i l}}}^{2}$ respectively. Similarly, compute $\beta_{y_{i l}}$ and $\sigma_{\beta_{y_{i l}}}^{2}$ values for all the experimental runs. Develop, then, the following regression model for prediction of $\beta_{y_{i l}}$ under an experimental run:

$$
\beta_{y_{i l}}=b_{0}+\sum_{u=1}^{q} b_{u} X_{u}+\sum_{u, v} b_{u v} X_{u} X_{v}+N\left(\delta_{0}+\sum_{u=1}^{q} \delta_{u} X_{u}\right)+\varepsilon
$$

and the following regression model for prediction of $\sigma_{\beta_{y_{i l}}}^{2}$ value:

$$
\log _{10}\left(\sigma_{\beta_{y_{i l}}^{2}}^{2}\right)=c_{0}+\sum_{u=1}^{q} c_{u} X_{u}+\sum_{u, v} c_{u v} X_{u} X_{v}+N\left(\delta_{0}^{\prime}+\sum_{u=1}^{q} \delta_{u}^{\prime} X_{u}\right)+\varepsilon
$$

where $b_{u}, c_{u}, b_{u v}, \quad c_{u v}, \delta_{0}, \delta_{0}^{\prime}(u=1,2, \ldots, q ; v=1,2,3, \ldots, q)$ are regression coefficients, $X_{u} \quad(u=1,2, \ldots, q)$ are controllable variables.

It may be noted that corresponding to $t$ experimental runs $\times n$ noise levels, there are $t \times n$ values of $\beta_{y_{i l}}$ and $\sigma_{\beta_{y_{i l}}}^{2}$ for each response variable $Y_{i}(i=1,2, \ldots, p)$. Using these values, the coefficients of the regression models can be easily obtained using the procedure described earlier, and then, diagnostic checks for validating the regression models as described in section 4.1 .1 must be performed. If no serious violations of model assumptions are detected, the fitted regression equation can be assumed to be appropriate for prediction of the response variables. 
Suppose that finally established (fitted) regression equations for prediction of $\beta_{y_{i l}}$ and $\sigma_{\beta_{y_{i l}}}^{2}(i=1,2, \ldots, p ; l=1,2, \ldots, n)$ are as follows:

$$
\begin{aligned}
& \beta_{y_{i l}}=\hat{b}_{0}+\sum_{u=1}^{q} \hat{b}_{u} X_{u}+\sum_{u, v} \hat{b}_{u v} X_{u} X_{v}+N\left(\hat{\delta_{0}}+\sum_{u=1}^{q} \hat{\delta_{u}} X_{u}\right) \\
& \log _{10}\left(\sigma_{\beta_{y_{i l}}^{2}}^{2}\right)=\hat{c}_{0}+\sum_{u=1}^{q} \hat{c}_{u} X_{u}+\sum_{u, v} \hat{c}_{u v} X_{u} X_{v}+N\left(\hat{\delta_{0}^{\prime}}+\sum_{u=1}^{q} \hat{\delta_{u}^{\prime}} X_{u}\right)
\end{aligned}
$$

where $\hat{b}_{u}, \hat{c}_{u}, \hat{b}_{u v}, \hat{c}_{u v}, \hat{\delta}_{0}, \hat{\delta}_{u}^{\prime}(u=1,2, \ldots, q ; v=1,2,3, \ldots, q)$ are the estimates of the regression coefficients.

\subsubsection{Multiple regression equations for prediction under RM approach}

In RM approach, each response is directly modelled as function of the control, noise and signal factors. Let the value of a response variable $Y_{i}(i=1,2, \ldots, p)$ in the signal level $k(k=1,2, \ldots, s)$ and noise level $l(l=1,2, \ldots, n)$ under an experimental run is denoted as $y_{i k l}$. Develop now the following regression model for prediction of $y_{i k l}$ under an experimental run:

$$
\log _{10}\left(y_{i k l}\right)=b_{0}+\sum_{u=1}^{q} b_{u} X_{u}+\sum_{u, v} b_{u v} X_{u} X_{v}+M\left(\gamma_{0}+\sum_{u=1}^{q} \gamma_{u} X_{u}\right)+N\left(\delta_{0}+\sum_{u=1}^{q} \delta_{u} X_{u}\right)+\varepsilon
$$

where $b_{u}, b_{u v}, \gamma_{u}, \delta_{u}(u=1,2, \ldots, q ; v=1,2,3, \ldots, q)$ are regression coefficients, $X_{u}(u=1,2, \ldots, q)$ are controllable variables, $y_{i k l}$ is the value of $i^{t h}$ response variable in the $k^{\text {th }}$ signal level and $l^{\text {th }}$ noise level, and $\varepsilon$ is the error which is assumed to follow a normal distribution with mean value as zero and a constant variance $\sigma^{2}$. Generally, it is expected that random error will have increased variability at higher signal levels instead of having constant variability at all signal levels. With the aim to ensure that its effect be smaller, log transformed value of a response variable is considered as the dependent variable.

It may be noted that apart from the main factors, the quadratic terms of main factors may be included in the model when there is three or more number of levels in any control and signal factors. The interaction terms of controllable variables may also be included in the model. But, it is very important to include a few control $\times$ signal and control $\times$ noise interaction terms into the response model. If a few control $\times$ signal and control $\times$ noise interaction terms are not included in the response model, $\log _{10}\left(y_{i k l}\right)$ will be additive at different signal levels independent of combinations of control factors $\mathbf{X}$, which implies that intercept only will change but slopes of the controllable variables $\mathbf{X}$ will remain constant. As a result, it will not be possible to obtain desired predicted values of the response variables at different signal and noise levels.

Corresponding to $t$ experimental runs, $s$ signal levels, $n$ noise levels and $r$ replications, there are $t \times s \times n \times r$ values for each response variable. Using these values, the coefficients of the regression models can be easily obtained, and then, diagnostic checks for validating the regression models as described in section 4.1.1 must be performed. If no serious violations of model assumptions are detected, the fitted regression equation can be assumed to be appropriate for prediction of the response variables.

Suppose that finally established (fitted) regression equations for prediction of the response variable $Y_{i}(i=1,2, \ldots, p)$ at $k^{\text {th }}$ signal level and $l^{\text {th }}$ noise level are as follows:

$\log _{10}\left(y_{i k l}\right)=\hat{b}_{0}+\sum_{u=1}^{p} \hat{b}_{u} X_{u}+\sum_{u, v} \hat{b}_{u v} X_{u} X_{v}+M\left(\hat{\gamma}_{0}+\sum_{u=1}^{p} \hat{\gamma}_{u} X_{u}\right)+N\left(\hat{\delta_{0}}+\sum_{u=1}^{p} \hat{\delta_{u}} X_{u}\right)$

where $\hat{b}_{u}, \hat{b}_{u v}, \hat{\gamma}_{u}, \hat{\delta}_{u}(u=1,2, \ldots, q ; v=1,2,3, \ldots, q)$ are estimates of the regression coefficients.

\subsection{Formulating the objective function for optimization}

If PMM approach is used for modelling the dynamic system, the values of slope $\left(\beta_{y_{i}}\right)$ and variance $\left(\sigma_{\beta_{y_{i}}}^{2}\right)$ around the slope for a response variable $Y_{i}$ for a given control factor settings can be predicted using the Eqns. (7) and (8) respectively. So MRDSN for the response variable $Y_{i}$ at the given control factor settings can be obtained straightway from these predicted values using Eqn. (4), and the weighted MRDSN (WMRDSN) over all the response variables can be considered as the overall performance under the control factor settings. Thus, the objective function under the PMM approach can be defined as follows:

$$
\mathrm{WMRDSN}_{\mathrm{PMM}}=\sum_{i=1}^{p} w_{i} \times \operatorname{MRDSN}_{y_{i}}=\sum_{i=1}^{p} w_{i} \times\left[10 \log _{10}\left(\frac{\hat{\beta_{y_{i}}^{2}}}{\hat{\sigma}_{\beta_{y_{i}}}^{2}}\right)\right]
$$


where, $\quad w_{i}$ is the relative weights for the response variables and $\hat{\beta}_{y_{i}}$ and $\hat{\sigma}_{\beta_{y_{i}}}^{2}$ are predicted values of slope and variance obtained by Eqns. (7) and (8) respectively for the response variable $Y_{i}(i=1,2, \ldots, p)$ and $\sum_{i=1}^{p} w_{i}=1$. It is suggested to consider $w_{i}=1 / p$, if the relative importance of the response variables are unknown.

If RFM approach is used for modelling the dynamic system, the values of slope $\left(\beta_{y_{i l}}\right)$ and variance $\left(\sigma_{\beta_{y_{i l}}}^{2}\right)$ around the slope for a response variable $Y_{i}$ in the noise level $l$ under an experimental run, can be predicted using the Eqns. (11) and (12) respectively. So using these predicted values, the $\operatorname{MRDSN}_{y_{i l}}$ for the response variable $Y_{i}$ in the noise level $l$ under an experimental run can be obtained using Eqn. (4). The performance of the response variable $Y_{i}$ at the given control factor settings $\left(\mathrm{MRDSN}_{y_{i}}\right.$ ) can be measured as the average of $\mathrm{MRDSN}_{y_{i l}}$ values over all the noise levels under the control factor settings, and WMRDSN over all the response variables can be considered as the overall performance under the given control factor settings. Thus, the objective function under the RFM approach can be defined as follows:

$$
\begin{aligned}
\mathrm{WMRDSN}_{\mathrm{RFM}} & =\sum_{i=1}^{p} w_{i} \times \mathrm{MRDSN}_{y_{i}}=\sum_{i=1}^{p} w_{i} \times\left(\frac{1}{n} \sum_{l=1}^{n} \mathrm{MRDSN}_{y_{i l}}\right) \\
= & \sum_{i=1}^{p} w_{i} \times\left[\frac{1}{n} \sum_{l=1}^{n} 10 \log _{10}\left(\frac{\hat{\beta}_{y l}^{2}}{\hat{\sigma}_{\beta_{y i l}}^{2}}\right)\right]
\end{aligned}
$$

where, $\hat{\beta}_{y_{i l}}^{2}$ and $\hat{\sigma}_{\beta_{y i l}}^{2}$ are the predicted values of slope and variance for the $i^{\text {th }}$ response variable at $l^{\text {th }}$ noise level, obtained based on Eqns. (11) and (12) respectively.

If RM approach is used for modelling the dynamic system, the predicted values of a response variable $Y_{i}(i=1,2, \ldots, p)$ at $k^{\text {th }}(k=1,2, \ldots, s)$ level of signal factor and $l^{\text {th }}(l=1,2, \ldots, n)$ level of noise factor, i.e. $\hat{y}_{i k l}$ under a given control factor settings can be obtained using Eqn. (14). Using these predicted values, the slope $\left(\beta_{y_{i}}\right)$ and variance around slope $\left(\sigma_{\beta_{y_{i}}}^{2}\right)$ for the response variable $Y_{i}$ under the control factor settings can be obtained using Eqns. (2) and (3) respectively, and then $\mathrm{MRDSN}_{y_{i}}$ for the response variable $Y_{i}(i=1,2, \ldots, p)$ under the control factor settings can be obtained using Eqn. (4). Therefore, the weighted average of MRDSN $_{y_{i}}$ values over all the response variables may be considered as the overall performance under the control factor settings. Thus, the objective function under the RM approach can be defined as follows:

$$
\begin{aligned}
\mathrm{WMRDSN}_{\mathrm{RM}} & =\sum_{i=1}^{p} w_{i} \times \operatorname{MRDSN}_{y_{i}}=\sum_{i=1}^{p} w_{i} \times 10 \log _{10}\left(\frac{\hat{\beta_{y_{i}}^{2}}}{\hat{\sigma}_{\beta_{y_{i}}^{2}}^{2}}\right) \\
& =\sum_{i=1}^{p} w_{i} \times 10 \log _{10}\left[\hat{\beta_{y_{i}}^{2}} /\left\{\left(\frac{1}{s n-1}\right) \sum_{k=1}^{s} \sum_{l=1}^{n}\left(\hat{y}_{i k l}-\hat{\beta}_{y_{i}} M_{k}\right)^{2}\right\}\right]
\end{aligned}
$$

where $\hat{\beta}_{y_{i}}=\sum_{k=1}^{s} \sum_{l=1}^{n} \hat{y}_{i k l} M_{k} / \sum_{j=1}^{s} M_{k}^{2}$ and, $\hat{y}_{i k l}(i=1,2, \ldots, p ; k=1,2, \ldots, s ; l=1,2, \ldots, n)$ are the predicted values of response variable $Y_{i}$ at $k^{\text {th }}$ level of signal factor and $l^{\text {th }}$ level of noise factor obtained from Eqn. (14).

\subsection{Determining optimal values of the levels of the control factors}

It may be noted that WMRDSN is essentially a function of the control factors whatever modelling approach be used for prediction. Since higher MRDSN implies better quality, the aim is to determine the level values of the control factors that will maximize the WMRDSN value. One simple approach for determining the optimal control factor settings can be as follows: a) choose an arbitrary setting combination of control factor levels, and obtain the predicted values using the relevant fitted multiple regression equations under the chosen control factor settings b) obtain MRDSN values for all the response variables under the control factor settings and then obtain the WMRDSN value under the control factor settings, c) change the level values of the control factors, compare the resulting WMRDSN values, and find out the control factor settings that results in the maximum WMRDSN value. It has been observed that if all the computations are carried out in Excel worksheet, this enumerative search for finding the optimal level values of the control factors can be performed very effectively using the 'Solver' tool of Microsoft Excel package. It is a kind of 'what if' analysis which discovers the optimal value of a target cell by varying values in cells employed to 
compute the target cell. The 'Solver' instrument utilizes the generalized reduced gradient (GRG) technique for searching the optimal solution, contributed by Del Castillo and Montgomery (1993). Examples on usage of 'Solver' tool are available in Pal and Gauri (2010).

Whilst operating the 'Solver' instrument it is essential to identify the range of echelons for the input variables. In particular instances that one or more input variables take only discrete values, the integer restriction (IR) for those input variables requires specification. Sometimes, one may have to add an additional constraint to keep the slope of the dynamic nominal-the-best variable closer to its target slope value. Unless technically there is IR for a response variable, preferably, the optimization ought not to be limited to only the real experimental design settings since it possibly will show the way to a suboptimal solution. Since the WMRDSN metric is described established on regression models, the proposed technique can offer an optimal solution in the whole range of experimental area of the input controllable variables whilst employing the 'Solver' instrument of Microsoft Excel package.

\section{Analysis and Results}

Under the current research, there is no scope for collection of primary data from industry. Therefore, it is decided to analyse one set of secondary data, i.e. published data in the literature for illustration of implementation of the proposed procedure as well as for evaluation of the optimization performance of the proposed procedure under different modelling approaches for the dynamic system. Chang (2008) has illustrated application of his proposed data mining approach for optimizing multi-response dynamic systems using a case study adopted from Chang (2006). This case involves simultaneous optimization of three dynamic response variables named $Y_{1}, Y_{2}$ and $Y_{3}$. Among these, $Y_{1}$ is dynamic larger- the-better (DLTB) type, $Y_{2}$ is dynamic nominal-the-best (DNTB) type and $Y_{3}$ is dynamic smaller-the-better (DSTB) type variable. In this case, six control factors A - F, each at three levels $(1,2$, and 3$)$, were considered and arranged in a standard $L_{18}$ orthogonal array as shown in Table 2.

Table 2. Experimental design used by Chang (2006)

\begin{tabular}{|c|c|c|c|c|c|c|}
\hline \multirow{2}{*}{ Trial no. } & \multicolumn{7}{|c|}{ Factors and levels } \\
\cline { 2 - 7 } & $A$ & $B$ & $C$ & $D$ & $E$ & $F$ \\
\hline 1 & 1 & 1 & 1 & 1 & 1 & 1 \\
\hline 2 & 2 & 2 & 2 & 2 & 2 & 2 \\
\hline 3 & 3 & 3 & 3 & 3 & 3 & 3 \\
\hline 4 & 1 & 1 & 2 & 2 & 3 & 3 \\
\hline 5 & 2 & 2 & 3 & 3 & 1 & 1 \\
\hline 6 & 3 & 3 & 1 & 1 & 2 & 2 \\
\hline 7 & 1 & 2 & 1 & 3 & 2 & 3 \\
\hline 8 & 2 & 3 & 2 & 1 & 3 & 1 \\
\hline 9 & 3 & 1 & 3 & 2 & 1 & 2 \\
\hline 10 & 1 & 3 & 3 & 2 & 2 & 1 \\
\hline 11 & 2 & 1 & 1 & 3 & 3 & 2 \\
\hline 12 & 3 & 2 & 2 & 1 & 1 & 3 \\
\hline 13 & 1 & 2 & 3 & 1 & 3 & 2 \\
\hline 14 & 2 & 3 & 1 & 2 & 1 & 3 \\
\hline 15 & 3 & 1 & 2 & 3 & 2 & 1 \\
\hline 16 & 1 & 3 & 2 & 3 & 1 & 2 \\
\hline 17 & 2 & 1 & 3 & 1 & 2 & 3 \\
\hline 18 & 3 & 2 & 1 & 2 & 3 & 1 \\
\hline & & & & & & \\
\hline
\end{tabular}

The signal factor of the case had three levels named $M_{1}, M_{2}$ and $M_{3}$, the corresponding values of which were 10, 20 and 30 , respectively. Two levels $\left(N_{1}\right.$ and $N_{2}$ ) of noise factor settings were considered in this case. The specifications for the response variables and the experimental data of Chang (2006) are reproduced in Table 2 and Table 3 respectively. The experimental data of Chang (2006) are analysed here.

It is important to mention that the past researchers, Chang (2006) and Chang (2008), have analysed the same experimental data for illustrating their optimization methods and determined the optimal settings with respect to the actual experimental design settings. As highlighted in the previous section, since the optimization function (WMRDSN) in our proposed method is described established on regression models, it has the capability of offering an optimal solution in the range of the experimental area of the input controllable variables. However, with the aim to make the optimal solution and related results of our method comparable with that of the past researchers, it is decided to specify integer restrictions for the level values of the control factors while running 'Solver' tool for the optimization. 
Table 3.Specifications for the response variables specified by Chang (2006)

\begin{tabular}{|c|c|c|c|c|c|}
\hline \multirow{3}{*}{$\begin{array}{c}\text { Signal } \\
\text { levels }\end{array}$} & \multicolumn{5}{|c|}{ Responses } \\
\cline { 2 - 6 } & $Y_{1}$ (DLTB) & \multicolumn{3}{|c|}{$Y_{2}$ (DNTB) } & $Y_{3}$ (DSTB) \\
\hline$M_{1}$ & 55 & 7 & 10 & 13 & 3 \\
\hline$M_{2}$ & 110 & 14 & 20 & 26 & 6 \\
\hline$M_{3}$ & 165 & 21 & 30 & 39 & 9 \\
\hline
\end{tabular}

Table 4. Experimental Data of Chang (2006)

\begin{tabular}{|c|c|c|c|c|c|c|c|c|c|c|c|c|c|c|c|c|c|c|c|}
\hline \multirow{5}{*}{$\begin{array}{c}\text { Trial } \\
\text { no. } \\
1\end{array}$} & \multirow{4}{*}{ 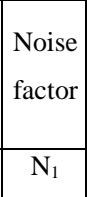 } & \multicolumn{18}{|c|}{ Responses } \\
\hline & & \multicolumn{6}{|c|}{$\mathrm{Y}_{1}$} & \multicolumn{6}{|c|}{$\mathrm{Y}_{2}$} & \multicolumn{6}{|c|}{$\mathrm{Y}_{3}$} \\
\hline & & \multicolumn{2}{|c|}{$\mathrm{M}_{1}=10$} & \multicolumn{2}{|c|}{$\mathrm{M}_{2}=20$} & \multicolumn{2}{|c|}{$\mathrm{M}_{3}=30$} & \multicolumn{2}{|c|}{$\mathrm{M}_{1}=10$} & \multicolumn{2}{|c|}{$\mathrm{M}_{2}=20$} & \multicolumn{2}{|c|}{$\mathrm{M}_{3}=30$} & \multicolumn{2}{|c|}{$\mathrm{M}_{1}=10$} & \multicolumn{2}{|c|}{$\mathrm{M}_{2}=20$} & \multicolumn{2}{|c|}{$\mathrm{M}_{3}=30$} \\
\hline & & 61.6 & 78.2 & 128.0 & 106.0 & 230.6 & 226.9 & 7.4 & 7.2 & 16.7 & 13.2 & 23.7 & 24.1 & 1.9 & 1.9 & 4.6 & & 7.6 & 4.6 \\
\hline & $\mathrm{N}_{2}$ & 70.8 & 57.1 & 137.3 & 160.3 & 282.2 & 252.5 & 9.1 & 10.2 & 22.8 & 17.8 & 26.2 & 26.7 & 2.0 & 2.1 & 3.9 & 4.8 & 4.7 & 4.3 \\
\hline \multirow{2}{*}{2} & $\mathrm{~N}_{1}$ & 88.3 & 93.6 & 175.2 & 181.5 & 259.7 & 304.5 & 10.1 & 8.8 & 23.4 & 22.6 & 29.6 & 30.3 & 1.8 & 2.0 & 4.0 & 2.8 & 6.0 & 3.3 \\
\hline & $\mathrm{N}_{2}$ & 72.9 & 72.7 & 174.0 & 145.5 & 258.4 & 214.6 & 8.7 & 9.2 & 19.1 & 24.1 & 31.4 & 30.5 & 1.9 & 2.2 & 3.8 & 3.5 & 6.7 & 6.1 \\
\hline \multirow{2}{*}{3} & $\mathrm{~N}_{1}$ & 80.8 & 81.1 & 154.3 & 157.4 & 238.1 & 237.8 & 10.8 & 10.5 & 20.1 & 20.9 & 30.6 & 32.4 & 1.0 & 3.2 & 4.4 & 5.4 & 8.1 & 6.7 \\
\hline & $\mathrm{N}_{2}$ & 77.2 & 83.3 & 167.1 & 159.0 & 251.8 & 257.9 & 10.6 & 10.9 & 21.8 & 23.7 & 30.5 & 32.7 & 1.4 & 2.6 & 3.8 & 3.9 & 8.0 & 2.7 \\
\hline \multirow{2}{*}{4} & $\mathrm{~N}_{1}$ & 65.9 & 71.3 & 179.2 & 151.5 & $\mid 196.1$ & 221.6 & 7.6 & 7.2 & 15.3 & 14.8 & 22.5 & 22.2 & 1.7 & 2.2 & 4.1 & 4.9 & 5.9 & 6.0 \\
\hline & $\mathrm{N}_{2}$ & 83.7 & 78.4 & 135.6 & 177.0 & 246.9 & 291.8 & 8.1 & 7.6 & 14.1 & 14.7 & 21.9 & 21.8 & 2.1 & 2.6 & 3.6 & 3.3 & 7.7 & 6.9 \\
\hline \multirow{2}{*}{5} & $\mathrm{~N}_{1}$ & 79.4 & 88.6 & 121.9 & 151.6 & 248.8 & 245.1 & 11.9 & 12.7 & 25.6 & 25.7 & 36.6 & 35.7 & 2.0 & 2.1 & 2.7 & 3.8 & 4.8 & 5.8 \\
\hline & $\mathrm{N}_{2}$ & 67.8 & 87.3 & 113.6 & 141.3 & 171.5 & 244.7 & 10.5 & 11.8 & 25.8 & 26.2 & 39.1 & 33.1 & 2.6 & 1.7 & 4.1 & 3.6 & 5.5 & 5.2 \\
\hline \multirow{2}{*}{6} & $\mathrm{~N}_{1}$ & 90.5 & 87.0 & 161.8 & 169.4 & 286.9 & 236.5 & 10.2 & 10.2 & 23.7 & 21.8 & 32.0 & 32.8 & 1.8 & 2.2 & 2.5 & 4.1 & 4.9 & 5.4 \\
\hline & $\mathrm{N}_{2}$ & 87.6 & 87.8 & 160.7 & 163.9 & 231.4 & 288.7 & 10.7 & 11.2 & 22.5 & 20.0 & 34.2 & 28.0 & 1.2 & 2.0 & 4.0 & 4.4 & 6.6 & 3.1 \\
\hline 7 & $\mathrm{~N}_{1}$ & 80.9 & 74.7 & 165.9 & 163.4 & 232.2 & 246.4 & 11.7 & $\mid 12.1$ & 23.3 & 23.3 & 33.5 & 32.7 & 1.9 & 1.6 & 5.2 & 5.1 & 6.6 & 5.3 \\
\hline & $\mathrm{N}_{2}$ & 69.9 & 78.7 & 141.7 & 159.1 & 260.4 & 239.7 & 11.6 & 11.6 & 22.0 & 22.5 & 33.8 & 34.0 & 1.9 & 1.7 & 3.6 & 5.1 & 5.8 & 5.1 \\
\hline 8 & $\mathrm{~N}_{1}$ & 92.3 & 71.7 & 185.7 & 154.3 & 233.1 & 240.5 & 8.3 & 8.4 & 16.9 & 18.5 & 28.1 & 27.3 & 2.8 & 1.5 & 3.4 & 3.4 & 6.2 & 3.7 \\
\hline & $\mathrm{N}_{2}$ & 104.8 & 89.4 & 216.1 & 173.0 & 340.6 & 308.9 & 8.2 & 6.1 & 18.5 & 15.1 & 29.1 & 21.1 & 1.8 & 2.2 & 4.1 & 4.3 & 9.0 & 5.0 \\
\hline 9 & $\mathrm{~N}_{1}$ & 92.8 & 59.8 & 130.7 & 142.2 & 257.3 & 266.0 & 8.6 & 9.7 & 17.3 & 18.4 & 30.9 & 34.5 & 2.3 & 1.7 & 4.5 & 5.1 & 7.4 & 6.5 \\
\hline & $\mathrm{N}_{2}$ & 82.1 & 87.0 & 175.1 & 138.2 & \begin{tabular}{|l|l}
161.4 \\
\end{tabular} & 274.4 & 8.2 & 8.4 & 18.6 & 18.0 & 31.3 & 30.9 & 2.2 & 1.2 & 4.0 & 4.0 & 7.2 & 6.7 \\
\hline 10 & $\mathrm{~N}_{1}$ & 86.0 & 100.0 & 179.1 & 175.4 & 246.9 & 244.9 & 6.9 & 6.6 & 15.8 & 16.4 & 22.2 & 24.9 & 1.7 & 1.7 & 3.9 & 5.8 & 5.2 & 8.4 \\
\hline & $\mathrm{N}_{2}$ & 81.9 & 91.2 & 190.8 & 137.8 & 293.5 & 227.1 & 8.3 & 7.2 & 14.3 & 16.6 & 23.1 & 24.2 & 2.1 & 1.8 & 3.9 & 4.8 & 4.5 & 8.0 \\
\hline 11 & $\mathrm{~N}_{1}$ & 76.3 & 78.2 & 140.0 & 154.7 & 264.1 & 260.1 & 9.5 & 9.2 & 27.9 & 25.4 & 32.3 & 33.8 & 1.0 & 1.7 & 4.1 & 4.0 & 3.9 & 5.6 \\
\hline 11 & $\mathrm{~N}_{2}$ & 67.1 & 76.0 & 169.5 & 175.4 & 239.2 & 251.8 & 10.8 & 12.2 & 23.6 & 20.4 & 26.8 & 29.2 & 2.1 & 2.3 & 5.0 & 4.6 & 6.7 & 4.5 \\
\hline 12 & $\mathrm{~N}_{1}$ & 91.4 & 81.8 & 160.1 & 167.2 & 238.5 & 197.2 & 11.8 & 10.5 & 22.9 & 25.9 & 36.9 & 36.8 & 2.1 & 1.9 & 4.0 & 3.7 & 7.4 & 6.2 \\
\hline & $\mathrm{N}_{2}$ & 85.1 & 63.8 & 123.8 & 166.4 & 233.5 & 242.0 & 11.6 & 11.5 & 22.1 & 22.6 & 32.5 & 31.9 & 1.6 & 0.9 & 4.2 & 4.1 & 2.1 & 6.9 \\
\hline 13 & $\mathrm{~N}_{1}$ & 87.9 & 82.5 & 146.5 & 167.0 & 212.4 & 222.2 & 10.1 & 10.3 & 19.9 & 22.2 & 27.1 & 27.7 & 2.0 & 2.3 & 5.0 & 4.7 & 6.7 & 7.3 \\
\hline 18 & $\mathrm{~N}_{2}$ & 57.4 & 78.5 & 91.6 & 182.3 & 250.3 & 207.3 & 10.6 & 10.1 & 20.1 & 19.2 & 27.8 & 24.6 & 2.2 & 2.1 & 4.5 & 4.7 & 7.4 & 6.2 \\
\hline 14 & $\mathrm{~N}_{1}$ & 88.1 & 78.1 & 156.7 & 170.1 & 239.1 & 215.2 & 12.1 & 10.0 & 24.1 & 20.2 & 28.0 & 35.6 & 1.7 & 2.1 & 3.8 & 3.3 & 7.2 & 4.9 \\
\hline & $\mathrm{N}_{2}$ & 81.7 & 75.7 & 140.2 & 127.8 & 241.4 & 211.7 & 11.3 & 11.8 & 23.3 & 24.4 & 32.1 & 39.9 & 1.8 & 2.7 & 3.1 & 3.8 & 4.9 & 4.7 \\
\hline 15 & $\mathrm{~N}_{1}$ & 101.8 & 78.2 & 168.3 & 180.8 & 240.6 & 235.2 & 10.2 & 7.6 & 14.9 & 19.4 & 26.9 & 19.5 & 1.7 & 2.2 & 5.3 & 3.6 & 5.0 & 7.5 \\
\hline 10 & $\mathrm{~N}_{2}$ & 80.4 & 76.5 & 206.7 & 222.6 & 325.1 & 285.2 & 8.7 & 7.2 & 16.8 & 14.1 & 26.6 & 25.1 & 2.2 & 1.9 & 4.8 & 2.6 & 3.8 & 5.5 \\
\hline 16 & $\mathrm{~N}_{1}$ & 77.4 & 75.4 & 171.7 & 159.0 & 201.3 & 219.7 & 10.4 & 10.6 & 20.7 & 22.2 & 34.4 & 30.0 & 1.9 & 2.3 & 3.1 & 4.2 & 5.8 & 3.3 \\
\hline & $\mathrm{N}_{2}$ & 72.0 & 69.5 & 189.1 & 168.6 & 254.3 & 237.3 & 11.1 & 10.9 & 20.6 & 21.4 & 30.5 & 31.4 & 2.1 & 1.6 & 4.1 & 4.8 & 5.4 & 6.9 \\
\hline & $\mathrm{N}_{1}$ & 71.4 & 69.2 & 145.0 & 152.5 & 223.8 & 218.7 & 8.8 & 8.4 & 19.0 & 13.8 & 26.2 & 24.1 & 1.5 & 2.1 & 3.8 & 4.4 & 6.9 & 4.2 \\
\hline & $\mathrm{N}_{2}$ & 77.0 & 70.5 & 158.4 & 154.0 & 218.4 & 224.1 & 9.2 & 9.0 & 16.7 & 17.4 & 27.1 & 26.2 & 1.6 & 1.8 & 3.7 & 4.4 & 4.6 & 4.2 \\
\hline 1 & $\mathrm{~N}_{1}$ & 82.8 & 67.8 & 183.7 & 175.5 & 276.1 & 254.4 & 10.7 & 9.2 & 19.8 & 20.1 & 27.3 & 31.0 & 2.5 & 2.2 & 3.1 & 3.4 & 7.7 & 7.4 \\
\hline & $\mathrm{N}_{2}$ & 85.2 & 92.0 & 154.4 & 157.6 & 249.3 & 286.1 & 11.3 & 7.7 & 19.3 & 22.6 & 29.4 & 24.6 & 1.6 & 1.8 & 3.9 & 4.7 & 6.0 & 7.3 \\
\hline
\end{tabular}




\subsection{Optimization using WMRDSN ${ }_{\mathrm{PMM}}$ as the objective function}

The values of slope $(\beta)$ and variance $\left(\sigma_{\beta}^{2}\right)$ around slope for each response variable in each experimental run are computed first from the experimental data using equations (2) and (3) respectively. These computed $\hat{\beta}$ and $\hat{\sigma}_{\beta}^{2}$ values for the three response variables corresponding to the 18 experimental runs are shown in Table 5.

Table 5. Computed $\hat{\beta}$ and $\hat{\sigma}_{\beta}^{2}$ values for the three response variables in 18 runs

\begin{tabular}{|c|r|r|r|r|r|r|}
\hline Expt. No. & \multicolumn{1}{|c|}{$\hat{\beta}_{y_{1}}$} & \multicolumn{1}{c|}{$\hat{\sigma}_{\beta_{y_{1}}}^{2}$} & \multicolumn{1}{c|}{$\hat{\beta}_{y_{2}}$} & $\hat{\sigma}_{\beta_{y_{2}}}^{2}$ & $\hat{\beta}_{y_{3}}$ & $\hat{\sigma}_{\beta_{y_{3}}}^{2}$ \\
\hline 1 & 76.920 & 642.9 & 8.518 & 5.64 & 1.884 & 0.861 \\
\hline 2 & 85.562 & 477.9 & 10.368 & 3.15 & 1.829 & 0.724 \\
\hline 3 & 81.336 & 43.6 & 10.614 & 1.12 & 2.138 & 2.187 \\
\hline 4 & 79.555 & 600.1 & 7.384 & 0.15 & 2.141 & 0.415 \\
\hline 5 & 73.396 & 607.0 & 12.268 & 2.79 & 1.798 & 0.223 \\
\hline 6 & 85.625 & 297.3 & 10.702 & 2.83 & 1.736 & 0.870 \\
\hline 7 & 80.366 & 93.2 & 11.271 & 0.35 & 2.027 & 0.541 \\
\hline 8 & 92.602 & 995.9 & 8.675 & 4.97 & 1.971 & 1.552 \\
\hline 9 & 78.061 & 966.3 & 9.880 & 2.58 & 2.250 & 0.269 \\
\hline 10 & 85.045 & 389.4 & 7.829 & 0.96 & 2.186 & 1.350 \\
\hline 11 & 82.543 & 156.1 & 10.761 & 9.93 & 1.869 & 0.808 \\
\hline 12 & 76.620 & 278.7 & 11.548 & 2.92 & 1.898 & 1.725 \\
\hline 13 & 74.245 & 580.7 & 9.384 & 2.95 & 2.307 & 0.115 \\
\hline 14 & 75.632 & 180.2 & 11.304 & 7.3 & 1.811 & 0.505 \\
\hline 15 & 92.000 & 757.9 & 8.186 & 5.26 & 1.893 & 1.121 \\
\hline 16 & 78.730 & 299.3 & 10.566 & 1.26 & 1.866 & 0.842 \\
\hline 17 & 74.338 & 22.7 & 8.571 & 1.87 & 1.773 & 0.662 \\
\hline 18 & 86.927 & 200.2 & 9.632 & 4.15 & 2.205 & 0.576 \\
\hline
\end{tabular}

Now using the models shown in Eqns. (5) and (6), the most appropriate multiple regression equations for prediction of slope $(\beta)$ and variance $\left(\sigma_{\beta}^{2}\right)$ around slope for the three response variables are established, as given below:

$$
\begin{aligned}
& \beta_{y_{1}}=53.810+2.732 A-9.048 B+21.202 C-4.877 D+23.905 E-3.984 F+2.255 B^{2}-4.983 C^{2} \\
& -4.567 E^{2}-0.997 C F+2.832 D F-1.470 E F \\
& {\left[R^{2}=0.994 \text { and adjusted } R^{2}=0.979\right]} \\
& \log _{10}\left(\sigma_{\beta_{y_{1}}}^{2}\right)=2.035+1.104 A+0.286 B-1.174 C+0.317 D+1.851 E-1.159 F-0.146 C^{2}-0.118 D^{2} \\
& -0.051 F^{2}+0.139 A C+0.121 A D-0.903 A E+0.168 C D+0.437 C F-0.099 D E \\
& {\left[R^{2}=0.999 \text { and adjusted } R^{2}=0.999\right]} \\
& \beta_{y_{2}}=5.090+3.260 A+5.850 B-2.730 C-2.235 D-2.863 E+2.973 F-0.698 A^{2}-1.329 B^{2} \\
& +0.607 C^{2}+0.689 D^{2}+0.557 E^{2}-0.627 F^{2} \\
& {\left[R^{2}=0.979 \text { and adjusted } R^{2}=0.928\right]} \\
& \log _{10}\left(\sigma_{\beta_{y_{2}}}^{2}\right)=0.827-0.668 A-0.074 B+0.360 C+1.124 D+1.160 E-1.885 F-0.196 A^{2}+0.214 A D \\
& +0.702 A F-0.087 B C+0.144 B F-0.300 C D-0.109 B E-0.461 D E \\
& {\left[R^{2}=0.999 \text { and adjusted } R^{2}=0.999\right]} \\
& \beta_{y_{3}}=2.223-0.538 A-0.453 B+0.527 C+0.884 D-1.048 E+0.124 F+0.182 A^{2}-0.155 D^{2} \\
& +0.167 E^{2}-0.118 A C+0.214 B E-0.095 C D-0.039 C F \\
& {\left[R^{2}=0.997 \text { and adjusted } R^{2}=0.986\right]}
\end{aligned}
$$




$$
\begin{gathered}
\log _{10}\left(\sigma_{\beta_{y_{3}}}^{2}\right)=0.556-0.208 A-0.197 B+1.221 C-1.291 D-0.394 E-0.048 F+0.148 B^{2}-0.294 C^{2} \\
+0.144 D^{2}-0.106 E^{2}+0.096 F^{2}+0.118 A E-0.114 B F+0.373 D E-0.084 E F \\
{\left[R^{2}=0.994 \quad \text { and adjusted } R^{2}=0.945\right]}
\end{gathered}
$$

The existing setting combination of control factors $\mathrm{A}_{2} \mathrm{~B}_{2} \mathrm{C}_{2} \mathrm{D}_{2} \mathrm{E}_{2} \mathrm{~F}_{2}$ is chosen and slope and variance around the slope values for all the three response variables under the chosen setting combination are predicted using Eqns. (18-23). Then, WMRDSN $_{\text {PMM }}$ value under the chosen setting combination is computed using Eqn. (15). All these computations are carried out in Excel worksheet, and using 'Solver' tool of Microsoft Excel package WMRDSN ${ }_{\text {PMM }}$ is maximized. It is found that the value

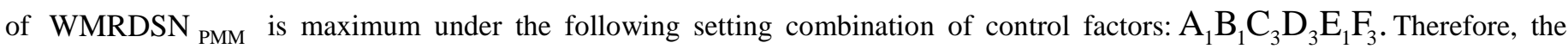
optimal solution under the proposed procedure with $P M M$ approach is chosen as $A_{1} B_{1} C_{3} D_{3} E_{1} F_{3}$. The expected slope and variance values, and the MRDSN values for each response variable at this optimal combination are shown in Table 6.

Table 6: Expected slope, variance and MRDSN at the PMM-based optimal condition

\begin{tabular}{|c|c|c|c|}
\hline Response Variable & Slope & Variance & MRDSN \\
\hline$Y_{1}$ (DLTB) & 73.368 & 25.930 & 23.172 \\
\hline$Y_{2}$ (DNTB) & 9.893 & 0.037 & 34.219 \\
\hline$Y_{3}$ (DSTB) & 2.397 & 0.394 & 11.638 \\
\hline \multicolumn{2}{|r}{} & WMRDSN & 23.009 \\
\hline
\end{tabular}

\subsection{Optimization using WMRDSN ${ }_{\mathrm{RFM}}$ as the objective function}

The values slope $(\beta)$ and variance $\left(\sigma_{\beta}^{2}\right)$ around slope in each noise level under all the experimental run for $p$ response variables are computed. These computed values for the three response variables are recorded in the format shown in Table 7.

Table 7. Computed $\hat{\beta}$ and $\hat{\sigma}_{\beta}^{2}$ values for the responses in different noise levels of the runs

\begin{tabular}{|c|c|c|c|c|c|c|c|}
\hline Expt. No. & Noise level & $\hat{\beta}_{y_{1 k}}$ & $\hat{\sigma}_{\beta_{y_{k}}}^{2}$ & $\hat{\beta}_{y_{2 k}}$ & $\hat{\sigma}_{\beta_{y_{2 k}}}^{2}$ & $\hat{\beta}_{y_{3 k}}$ & $\hat{\sigma}_{\beta_{y_{3 k}}^{2}}^{2}$ \\
\hline \multirow{2}{*}{1} & $N_{1}$ & 70.725 & 426.61 & 7.779 & 1.61 & 2.036 & 0.99 \\
\cline { 2 - 8 } & $N_{2}$ & 83.114 & 557.99 & 9.257 & 4.68 & 1.732 & 0.65 \\
\hline \multirow{2}{*}{2} & $N_{1}$ & 92.425 & 234.56 & 10.379 & 3.20 & 1.618 & 0.94 \\
\cline { 2 - 8 } & $N_{2}$ & 78.70 & 289.27 & 10.357 & 3.718 & 2.039 & 0.16 \\
\cline { 2 - 8 } & $N_{1}$ & 79.036 & 4.703 & 10.439 & 0.49 & 2.436 & 0.83 \\
\hline \multirow{3}{*}{4} & $N_{2}$ & 83.636 & 31.935 & 10.789 & 1.639 & 1.839 & 2.99 \\
\cline { 2 - 8 } & $N_{1}$ & 73.275 & 342.968 & 7.468 & 0.058 & 2.057 & 0.174 \\
\hline \multirow{2}{*}{5} & $N_{2}$ & 85.836 & 535.581 & 7.30 & 0.20 & 2.225 & 0.66 \\
\cline { 2 - 8 } & $N_{1}$ & 78.454 & 326.596 & 12.289 & 0.811 & 1.746 & 0.284 \\
\hline \multirow{2}{*}{6} & $N_{2}$ & 68.339 & 722.379 & 12.246 & 5.338 & 1.85 & 0.176 \\
\hline \multirow{2}{*}{7} & $N_{1}$ & 86.075 & 285.870 & 10.921 & 1.015 & 1.718 & 0.336 \\
\cline { 2 - 8 } & $N_{2}$ & 85.175 & 366.018 & 10.482 & 4.662 & 1.754 & 1.574 \\
\hline \multirow{2}{*}{8} & $N_{2}$ & 80.357 & 34.700 & 11.271 & 0.671 & 2.136 & 0.631 \\
\cline { 2 - 8 } & $N_{1}$ & 80.875 & 170.342 & 11.271 & 0.109 & 1.918 & 0.426 \\
\hline \multirow{2}{*}{9} & $N_{2}$ & 104.318 & 465.830 & 8.289 & 8.557 & 2.243 & 1.706 \\
\cline { 2 - 8 } & $N_{1}$ & 81.011 & 538.779 & 9.889 & 2.923 & 2.318 & 0.204 \\
\hline
\end{tabular}


Table 7 (cont'd). Computed $\hat{\beta}$ and $\hat{\sigma}_{\beta}^{2}$ values for the responses in different noise levels of the runs

\begin{tabular}{|c|c|c|c|c|c|c|c|}
\hline Expt. No. & Noise level & $\hat{\beta}_{y_{1 k}}$ & $\hat{\sigma}_{\beta_{y_{k}}}^{2}$ & $\hat{\beta}_{y_{2 k}}$ & $\hat{\sigma}_{\beta_{y_{2 k}}}^{2}$ & $\hat{\beta}_{y_{3 k}}$ & $\hat{\sigma}_{\beta_{y_{3 k}}}^{2}$ \\
\hline \multirow{2}{*}{10} & $N_{1}$ & 84.657 & 100.460 & 7.829 & 1.319 & 2.271 & 1.553 \\
\cline { 2 - 8 } & $N_{2}$ & 85.432 & 754.592 & 7.829 & 0.801 & 2.10 & 1.334 \\
\hline \multirow{2}{*}{11} & $N_{1}$ & 82.732 & 244.166 & 11.557 & 8.86 & 1.693 & 0.606 \\
\cline { 2 - 8 } & $N_{2}$ & 82.354 & 98.92 & 9.964 & 5.889 & 2.043 & 0.830 \\
\cline { 2 - 8 } & $N_{1}$ & 76.246 & 324.928 & 12.179 & 1.533 & 2.150 & 0.296 \\
\hline \multirow{2}{*}{13} & $N_{2}$ & 76.993 & 286.58 & 10.918 & 0.45 & 1.646 & 2.788 \\
\cline { 2 - 8 } & $N_{1}$ & 75.043 & 138.092 & 9.607 & 2.866 & 2.346 & 0.08 \\
\hline \multirow{2}{*}{14} & $N_{2}$ & 73.446 & 1132.33 & 9.161 & 3.059 & 2.268 & 0.156 \\
\cline { 2 - 8 } & $N_{1}$ & 77.954 & 136.142 & 10.768 & 8.022 & 1.939 & 0.635 \\
\hline \multirow{2}{*}{15} & $N_{2}$ & 73.311 & 200.041 & 11.839 & 4.815 & 1.682 & 0.290 \\
\hline \multirow{2}{*}{16} & $N_{1}$ & 82.343 & 169.944 & 8.057 & 9.268 & 2.114 & 0.973 \\
\cline { 2 - 8 } & $N_{2}$ & 101.657 & 452.844 & 8.314 & 2.117 & 1.671 & 0.943 \\
\hline \multirow{2}{*}{17} & $N_{1}$ & 74.186 & 225.805 & 10.714 & 2.185 & 1.646 & 0.96 \\
\cline { 2 - 8 } & $N_{2}$ & 83.275 & 201.30 & 10.418 & 0.33 & 2.086 & 0.357 \\
\hline \multirow{2}{*}{18} & $N_{1}$ & 73.682 & 14.07 & 8.346 & 3.226 & 1.904 & 0.85 \\
\cline { 2 - 8 } & $N_{2}$ & 74.993 & 31.036 & 8.796 & 0.32 & 1.643 & 0.416 \\
\hline
\end{tabular}

Now using the models shown in Eqns. (9) and (10), the most appropriate multiple regression equations for prediction of slope ( $\beta$ ) and variance around slope $\left(\sigma_{\beta}^{2}\right)$ for the three response variables are established, as given below:

$$
\begin{aligned}
& \beta_{y_{1}}= 52.9+2.36 A-8.30 B+18.74 C-4.13 D+20.6 E-1.34 F+1.53 N+2.13 B^{2}-4.86 C^{2} \\
&-4.76 E^{2}+2.62 D F-1.74 E F+2.93 E N-2.44 F N \\
& {\left[R^{2}=0.642 \text { and adjusted } R^{2}=0.404\right] } \\
& \log _{10}\left(\sigma_{\beta_{y_{1}}}^{2}\right)=2.534+1.791 A+0.016 B-1.896 C+1.125 D+1.78 E-2.027 F-0.437 N-0.18 D^{2} \\
&+0.174 A B-1.155 A E-0.074 B C+0.696 C F-0.0585 A N+0.1 B N+.231 C N+0.105 E N \\
& \quad\left[R^{2}=0.803 \text { and adjusted } R^{2}=0.595\right] \\
& \beta_{y_{2}=} 3.71+3.651 A+4.976 B-2.789 C-1.414 D-1.586 E+2.16 F+0.89 N-0.698 A^{2}-1.248 B^{2}+0.624 C^{2} \\
&+0.647 D^{2}+0.506 E^{2}-0.564 F^{2}+0.256 A B-0.237 A D-0.272 A E+0.184 A F-0.169 A N-0.326 E N \\
& \quad\left[R^{2}=0.949 \text { and adjusted } R^{2}=0.889\right] \\
& \log _{10}\left(\sigma_{\beta_{y_{2}}}^{2}\right)=-0.37+2.407 A-0.436 B-0.827 C-0.022 D-0.046 E+0.162 F-0.117 N-0.48 A^{2}+0.2 C^{2} \\
&-0.217 F^{2}-0.273 A B-0.094 B E+0.467 B F+0.118 A N+0.156 B N-0.148 D N+0.245 E N-0.3 F N \\
& {\left[R^{2}=0.803 \text { and adjusted } R^{2}=0.595\right] } \\
& \beta_{y_{3}}=1.848-0.649 A-0.178 B+0.278 C+0.913 D-0.782 E+0.147 F+0.265 N+0.206 A^{2} \\
&-0.165 D^{2}+0.133 E^{2}+0.099 A E-0.113 C D-0.131 A N+0.0795 E N-0.109 F N \\
& {\left[R^{2}=0.631 \text { and adjusted } R^{2}=0.355\right] }
\end{aligned}
$$




$$
\begin{aligned}
& \log _{10}\left(\sigma_{\beta_{\gamma_{3}}}^{2}\right)=1.91+1.857 A-1.344 B-0.916 C+0.949 D-1.485 E-1.195 F-0.522 N-0.154 A^{2}+0.256 B^{2}+0.177 F^{2} \\
&-0.669 A D+0.087 B D+0.55 C E+0.313 D E-0.095 E F+0.162 A N-0.154 D N+0.115 E N+0.16 F N \\
& {\left[R^{2}=0.735 \text { and adjusted } R^{2}=0.42\right] }
\end{aligned}
$$

An arbitrary setting combination of control factors is chosen as $A_{2} B_{2} C_{2} D_{2} E_{2} F_{2}$ and slope and variance around the slope values for all the three response variables in both the noise levels under the chosen setting combination are predicted using Eqns. (24-29). Then, WMRDSN ${ }_{\text {RFM }}$ values under the chosen setting combination are computed using Eqn. (16). All these computations are carried out in Excel worksheet, and using 'Solver' tool of Microsoft Excel package WMRDSN ${ }_{\text {RFM }}$ is maximized. It is found that the optimal setting combination of control factors is $\mathrm{A}_{3} \mathrm{~B}_{1} \mathrm{C}_{1} \mathrm{D}_{3} \mathrm{E}_{2} \mathrm{~F}_{3}$. The expected slope and variance values, and the MRDSN

\begin{tabular}{|c|c|c|c|c|c|c|c|}
\hline $\begin{array}{c}\text { Response } \\
\text { Variable }\end{array}$ & $\begin{array}{l}\text { Noise } \\
\text { level }\end{array}$ & Slope & Variance & MRDSN & $\begin{array}{l}\text { Average } \\
\text { Slope }\end{array}$ & $\begin{array}{l}\text { Average } \\
\text { Variance }\end{array}$ & $\begin{array}{l}\text { Average } \\
\text { MRDSN }\end{array}$ \\
\hline \multirow{2}{*}{$Y_{1}(\mathrm{DLTB})$} & $N_{1}$ & 86.14 & 6.252 & 30.744 & \multirow[b]{2}{*}{86.175} & \multirow[b]{2}{*}{5.785} & \multirow[b]{2}{*}{31.085} \\
\hline & $\mathrm{N}_{2}$ & 86.21 & 5.317 & 31.454 & & & \\
\hline \multirow{2}{*}{$Y_{2}(\mathrm{DNTB})$} & $N_{1}$ & 10.171 & 0.589 & 22.447 & \multirow[b]{2}{*}{10.036} & \multirow[b]{2}{*}{0.395} & \multirow[b]{2}{*}{24.067} \\
\hline & $\mathrm{N}_{2}$ & 9.902 & 0.201 & 26.884 & & & \\
\hline \multirow{2}{*}{$Y_{3}(\mathrm{DSTB})$} & $N_{1}$ & 2.080 & 0.069 & 18.000 & \multirow[b]{2}{*}{1.932} & \multirow[b]{2}{*}{0.090} & \multirow[b]{2}{*}{16.173} \\
\hline & $N_{2}$ & 1.785 & 0.112 & 14.552 & & & \\
\hline & & & & & & WMRDSN & 23.775 \\
\hline
\end{tabular}
values for each response variable at this optimal combination under different noise levels are estimated and given in Table 8 .

Table 8: Expected slope, variance and MRDSN at the RFM-based optimal condition

\subsection{Optimization using WMRDSN ${ }_{\mathrm{RM}}$ as the objective function}

Using the model shown in Eqn. (13), the most appropriate multiple regression equations for prediction of values of the three response variables corresponding to different control factor settings, noise levels and signal levels are established, as given below: $\log _{10}\left(y_{1}\right)=10.1489+0.01338 A+0.0450 B+0.1216 C+0.0042 D+0.0958 E+0.0021 F+0.5084 M$

$$
\begin{aligned}
& -0.0562 M^{2}+0.0253 N-0.0243 C^{2}-0.0249 E^{2}-0.0098 M B-0.0097 M C-0.008 N B \\
& -0.0068 N C+0.0126 N E-0.01 N F \\
& {\left[R^{2}=0.943 \text { and adjusted } R^{2}=0.938\right]}
\end{aligned}
$$

$$
\begin{aligned}
\log _{10}\left(y_{2}\right)= & 0.2784+0.1258 A+0.2846 B-0.1459 C-0.1315 D-0.1074 E+0.1531 F+0.5486 M \\
& -0.0771 M^{2}+0.0273 N-0.0266 A^{2}-0.0651 B^{2}+0.0324 C^{2}+0.0388 D^{2}+0.0259 E^{2} \\
& -0.0323 F^{2}-0.0137 N E \\
& {\left[R^{2}=0.967 \text { and adjusted } R^{2}=0.965\right] } \\
\log _{10}\left(y_{3}\right)= & -0.2005-0.0729 A-0.0038 B+0.0191 C+0.0626 D-0.1014 E+0.0304 F+0.5667 M \\
& -0.0905 M^{2}+0.0689 N+0.0274 A^{2}-0.0207 D^{2}+0.0176 E^{2}+0.0077 M C+0.0085 M E \\
& -0.03334 N A-0.0154 N C+0.0152 N D+0.0194 N E-0.0242 N F \\
& {\left[R^{2}=0.822 \text { and adjusted } R^{2}=0.804\right] }
\end{aligned}
$$

An arbitrary setting combination of control factor is chosen as $A_{2} B_{2} C_{2} D_{2} E_{2} F_{2}$. For this setting combination, the predicted values of the three response variables at each of three signal levels and two noise levels are obtained using Eqns. (30)-(32). Then, WMRDSN $_{\mathrm{RM}}$ value under the chosen setting combination is obtained using Eqn. (17). Using 'Solver' tool in Excel worksheet, WMRDSN $_{\mathrm{RM}}$ value is maximized by changing values of input controllable variables. The optimal setting combination is found to be $A_{2} B_{1} C_{3} D_{3} E_{2} F_{2}$. The expected values of each response variable at the optimal combination and the corresponding slope, variance and MRDSN values are shown in Table 9. 
Table 9. Expected slope, variance and MRDSN values at RM-based optimal solution

\begin{tabular}{|c|c|c|c|c|c|c|c|}
\hline \multirow{2}{*}{$\begin{array}{l}\text { Response } \\
\text { variable }\end{array}$} & \multirow{2}{*}{$\begin{array}{l}\text { Noise } \\
\text { level }\end{array}$} & \multicolumn{3}{|c|}{ Expected values of the responses } & \multicolumn{3}{|c|}{ Expected } \\
\hline & & Signal $\mathrm{M}_{1}$ & Signal $\mathrm{M}_{2}$ & Signal $\mathrm{M}_{3}$ & Slope & Variance & MRDSN \\
\hline \multirow{2}{*}{$Y_{1}(\mathrm{DLTB})$} & $N_{1}$ & 77.797 & 155.546 & 240.082 & \multirow{2}{*}{79.416} & \multirow{2}{*}{6.745} & \multirow{2}{*}{29.709} \\
\hline & $N_{2}$ & 78.174 & 156.300 & 241.246 & & & \\
\hline \multirow{2}{*}{$Y_{2}(\mathrm{DNTB})$} & $N_{1}$ & 9.749 & 20.241 & 29.467 & \multirow{2}{*}{9.902} & \multirow{2}{*}{0.109} & \multirow{2}{*}{29.551} \\
\hline & $N_{2}$ & 9.747 & 20.239 & 29.463 & & & \\
\hline \multirow{2}{*}{$Y_{3}(\mathrm{DSTB})$} & $N_{1}$ & 1.799 & 3.894 & 5.555 & \multirow{2}{*}{1.858} & \multirow{2}{*}{0.014} & \multirow{2}{*}{23.947} \\
\hline & $N_{2}$ & 1.767 & 3.824 & 5.455 & & & \\
\hline & & & & & & WMRDSN & 27.736 \\
\hline
\end{tabular}

\subsection{Comparison of Optimization Performance of the Proposed Method under different modelling approaches for Dynamic} System:

The overall optimization performance of the proposed method under the three modelling approaches for dynamic system is summarized in Table 10. Table 10 reveals that the optimal solution derived by the proposed method depends on the choice of the modelling approach for the dynamic system. Here, the proposed method under the three modelling approaches results in three different optimal solutions. Comparison of WMRDSN values in Table 10, the optimal solution derived by the proposed approach with $\mathrm{RM}$ approach, i.e. $\mathrm{A}_{2} \mathrm{~B}_{1} \mathrm{C}_{3} \mathrm{D}_{3} \mathrm{E}_{2} \mathrm{~F}_{2}$ results in the maximum WMRDSN value. Moreover, it is observed from Eqns. (30-32) that $R^{2}$ values of all these fitted models are very high, implying that derived optimal solutions based on these fitted models would be highly reliable. This implies that the proposed method with RM approach leads to the best optimization performance. On the other hand, proposed method with PMM and RFM approaches lead to poor WMRDSN values. However, in our opinion, the proposed method with RFM approach is more undesirable than the proposed method with PMM approach. This is because regression models (Eqns. 24-29) fitted in RFM approach have lower $\mathrm{R}^{2}$ values, and hence, they are less reliable for predictive purpose. We could not explain the reasons for lower $\mathrm{R}^{2}$ values for the regression models fitted in RFM approach.

Table 10. Optimization performance of the proposed method under different modelling approaches

\begin{tabular}{|c|c|c|c|c|c|}
\hline \multirow{2}{*}{$\begin{array}{c}\text { Modelling } \\
\text { approach }\end{array}$} & Optimal solution & \multicolumn{3}{|c|}{ Expected MRDSN values for } & \multirow{2}{*}{ WMRDSN } \\
\cline { 3 - 5 } & & $Y_{1}$ (DLTB) & $Y_{2}$ (DNTB) & $Y_{3}$ (DSTB) & \\
\hline PMM & $\mathrm{A}_{1} \mathrm{~B}_{1} \mathrm{C}_{3} \mathrm{D}_{3} \mathrm{E}_{1} \mathrm{~F}_{3}$ & 23.172 & 34.219 & 11.638 & 23.009 \\
\hline RFM & $\mathrm{A}_{3} \mathrm{~B}_{1} \mathrm{C}_{1} \mathrm{D}_{3} \mathrm{E}_{2} \mathrm{~F}_{3}$ & 31.085 & 24.067 & 16.173 & 23.775 \\
\hline RM & $\mathrm{A}_{2} \mathrm{~B}_{1} \mathrm{C}_{3} \mathrm{D}_{3} \mathrm{E}_{2} \mathrm{~F}_{2}$ & 29.709 & 29.551 & 23.947 & 27.736 \\
\hline
\end{tabular}

It is important to mention that examining $R^{2}$ values of the fitted models under different modelling approaches may not be the only criterion for choosing the most appropriate modelling approach. The choice of the modelling approach to be followed often depends on the experimental data. For example, if there is a single observation (i.e. absence of noise factors) for each response variable in an experimental trial, the RFM approach cannot be applied. If the effect of noise variables on any response is relatively high, which can be easily identified from high variation of observations within a signal level, the PMM approach may lead to erroneous result. However, if it is possible to develop response models with very good fit (i.e. adjusted $\mathrm{R}^{2}>0.9$ ) and the fitted models can reproduce the experimental data with minimum variation, then such response models would always be the best choice for finding the optimal combination. However, as mentioned earlier, response models must include few control $\times$ signal and control $\times$ noise interaction terms apart from the main effects of control, signal and noise variables. Diagnostic tests related to regression model fitting must be carried out and validated. If model assumptions are violated, the corresponding models should not be used for optimization purpose.

\subsection{Comparison of optimization performance of the proposed method with response modelling approach and some reported methods}

Chang (2006) and Chang (2008) have used the same experimental data to illustrate the usefulness of back propagation neural network-based multi-response optimization method and data mining approach-based multi-response optimization method respectively. Chang (2006) derived the optimal combination of factor levels as $A_{3} B_{3} C_{1} D_{3} E_{2} F_{1}$, whereas Chang (2008) obtained the optimal process condition as $\mathrm{A}_{1} \mathrm{~B}_{3} \mathrm{C}_{1} \mathrm{D}_{3} \mathrm{E}_{2} \mathrm{~F}_{1}$. On the other hand, according to the proposed WMRDSN method with response modelling the optimal process condition is derived as $A_{2} B_{1} C_{3} D_{3} E_{2} F_{2}$. Expected total DSN values under these three optimal conditions are obtained. These values are presented in Table 11. It is observed from Table 11 that the expected total DSN value at the optimal condition derived by the proposed WMRDSN method with response modelling is much higher than the expected total DSN values at the optimal conditions derived by the existing neural network approach (2006) and data mining approach (2008). This is indicative that the proposed WMRDSN method with response modelling results in better optimization performance than the back propagation neural network-based approach and data mining-based approach. 
Table 11. Expected total DSN values at the optimal conditions derived by the proposed method and some existing methods

\begin{tabular}{|c|c|c|c|c|c|}
\hline \multirow{2}{*}{ Optimization Method } & \multirow{2}{*}{ Optimal Solution } & \multicolumn{3}{|c|}{ DSN (in dB) } & \multirow{2}{*}{$\begin{array}{l}\text { Total } \\
\text { DSN }\end{array}$} \\
\hline & & $Y_{1}$ & $Y_{2}$ & $Y_{3}$ & \\
\hline $\begin{array}{l}\text { Proposed WMRDSN method } \\
\text { with response modelling }\end{array}$ & $\mathrm{A}_{2} \mathrm{~B}_{1} \mathrm{C}_{3} \mathrm{D}_{3} \mathrm{E}_{2} \mathrm{~F}_{2}$ & 29.709 & 29.551 & 23.947 & 83.207 \\
\hline $\begin{array}{l}\text { Back propagation neural } \\
\text { network approach (2006) }\end{array}$ & $\mathrm{A}_{3} \mathrm{~B}_{3} \mathrm{C}_{1} \mathrm{D}_{3} \mathrm{E}_{2} \mathrm{~F}_{1}$ & 27.707 & 29.551 & 20.057 & 77.315 \\
\hline Data Mining Approach (2008) & $\mathrm{A}_{1} \mathrm{~B}_{3} \mathrm{C}_{1} \mathrm{D}_{3} \mathrm{E}_{2} \mathrm{~F}_{1}$ & 27.707 & 29.551 & 12.500 & 69.758 \\
\hline
\end{tabular}

\section{Conclusions}

In a multi-response dynamic system, the target values of the response variables depend on the setting of the signal factor. The majority of the contemporary mechanized processes utilise more than a few response variables and industries demand for developing procedures for optimizing multi-response dynamic system. To deal with the requirements of the contemporary industries, more than a few procedures for optimizing a multi-response dynamic system have been developed by the past researchers. But the optimization performances of these procedures are not very satisfactory. In this paper, a novel procedure for optimizing a multi-response dynamic system is proposed. The proposed procedure integrates multiple regression (MR) technique and Taguchi's dynamic signal-to-noise ratio (DSN) concept. The performance of the proposed method is evaluated with respect to three commonly used modelling approaches for the dynamic systems, e.g. performance measure modelling (PMM), response measure modelling (RFM) and response modelling (RM). It is observed that the proposed integrated method with RM approach results in the best optimization performance. It also results in better optimization performance than back propagation neural network-based approach and data mining-based approach reported by the past researchers.

\section{Acknowledgement}

Many thanks to the referees for their advantageous comments and ideas that have significantly enhanced the substance and arrangement of this contribution

\section{References}

Bae, S.J. and Tsui, K.L., 2006. Analysis of dynamic robust design experiment with explicit \& hidden noise variables. Quality Technology \& Quantitative Management, Vol. 3, No. 1, pp. 55-75.

Bashiri M., Amiri, A. and Jalili, M., 2016. Taguchi design optimization using multivariate process capability index. Journal of Industrial and Systems Engineering. Vol. 9, No. 1, pp. 57-78.

Chang, H.H., 2006. Dynamic multi-response experiments by back propagation networks and desirability functions. Journal of the Chinese Institute of Industrial Engineers, Vol. 23, No. 4, pp. 280-288.

Chang, H.H., 2008. A data mining approach to dynamic multiple responses in Taguchi experimental design. Expert System with Applications, Vol. 35, No.3, pp. 1095-1103.

Chang, H.H. and Chen, Y.K., 2011. Neuro-genetic approach to optimize parameter design of dynamic multi-response experiments. Applied Soft Computing Journal, Vol.11, No. 1, pp. 436 - 442.

Dasgupta, T., Miller, A. and Wu, C.F.J., 2010. Robust design of measurement systems, Technometrics, Vol. 52, No. 1, pp. 80-93.

Del Castillo, E. and Montgomery, D.C., 1993. A nonlinear programming solution to the dual response problem. Journal of Quality Technology, Vol. 25, No. 3, pp. 199-204.

Deng, J., 1982. Control problems of grey systems. Systems and Control Letters, Vol. 1, No. 5, pp. 288-294.

Derringer, G., and Suich, R., 1980. Simultaneous optimization of several response variables. Journal of Quality Technology, Vol. 12, No. 4, pp. 214-219.

Gauri, S.K., 2014. Optimization of multi-response dynamic systems using principal component analysis (PCA)-based utility theory approach. International Journal of Industrial Engineering Computations, Vol. 5, No.1, pp. 101-114.

He, Z., Zhou, P., Zhang, M and Goh, T.N., 2015. A review of analysis of dynamic response in design of experiments. Quality and Reliability Engineering International, Vol. 31, pp. 535-542.

Hsieh, K.L., Tong, L.I., Chiu, H.P. and Yeh, H.Y., 2005. Optimization of a multi-response problem in Taguchi's dynamic system. Computers and Industrial Engineering, Vol. 49, No. 4, pp. 556-571.

Hwang, C.L. and Yoon, K., 1981. Multiple Attribute Decision Making: Methods and Applications, Springer-Verlag, New York, USA.

Hwang, C.L., Lai, Y.J. and Liu, T.Y., 1993. A new approach for multiple objective decision making. Computers and Operational Research, Vol. 20, No. 9, pp. 889-899.

Joseph, V.R., and Wu, C.F.J. 2002. Robust parameter design of multiple-target systems. Technometrics, Vol. 44, No. 4, pp. 338346. 
Jung, J-R and Yum, B-J., 2011. Artificial neural network based approach for dynamic parameter design. Expert Systems with Applications, Vol. 38, pp. 504-510.

Lesperance, M.L., and Park, S.M., 2003. GLMs for the analysis of robust designs with dynamic characteristics. Journal of Quality Technology, Vol. 35, No. 3, pp. 253-263.

Lunani, M., Nair, V.N. and Wasserman, G.S., 1997. Graphical methods for robust design with dynamic characteristics. Journal of Quality Technology, Vol. 29, pp. 327-338.

Ma, Y-Z, Wang, J. and Su, G-J., 2012. Robust parameter design for dynamic multi-response system. System Engineering - Theory and Practice, Vol. 32, No. 8, pp.1841-1849.

McCaskey, S.D. and Tsui, K.L., 1997. Analysis of dynamic robust design experiments. International Journal of Production Research, Vol. 35, No. 6, pp. 1561-1574.

Miller, A. and Wu, C.F.J., 1996. Parameter design for signal-response systems: A different look at Taguchi's dynamic parameter design. Statistical Science, Vol. 11, No. 2, pp. 122-136.

Montgomery, D.C., Peck, E.A. and Vining, G.G., 2012. Introduction to Linear Regression Analysis, 5th ed., Wiley, New York.

Pal, S. and Gauri, S.K., 2010. Multi-response optimization using multiple regression based weighted signal-to-noise ratio (MRWSN). Quality Engineering, Vol. 22, No. 4, pp. 336-350.

Sharma, N.K., Cudney, E.A. and Corns, S.M., 2012. Integration of dynamic multi-response systems using the product of normalised squared-bias and variance. International Journal of Quality Engineering and Technology, Vol.3, No.2, pp.108 - 123.

$\mathrm{Su}, \mathrm{C}-\mathrm{T}$., Chen, M-C., and Chan, H-L., 2005. Applying neural network and scatter search to optimize parameter design with dynamic characteristics. Journal of Operations Research Society, Vol. 56, No. 10, pp. 1132-1140.

Taguchi, G., 1990. Introduction to Quality Engineering, Asian Productivity Organization, Tokyo, Japan.

Tong, L.I., Wang, C.H., Houng, J.Y. and Chen, J.Y. 2002. Optimizing dynamic multi-response problems using the dual-responsesurface method. Quality Engineering, Vol. 14, No. 1, PP. 115-125.

Tong, L.I., Wang, C.H., Chen, C.C. and Chen, C.T., 2004. Dynamic multiple responses by ideal solution analysis. European Journal of Operations Research, Vol. 156, No. 2, pp. 433-441.

Tong, L.I., Wang, C.H. and Tsai, C.W., 2008. Robust design for multiple dynamic quality characteristics using data envelopment analysis. Quality and Reliability Engineering International, Vol. 24, No. 5, pp. 557-571.

Tsui, K., 1998. Alternatives of Taguchi's Approach for Dynamic Robust design Problems. International Journal of Reliability, Quality and Safety Engineering, Vol. 5, No. 2, pp. 115-131.

Tsui, K., 1999. Modelling and analysis of dynamic robust design experiments. IIE Transactions, Vol. 31, No. 12, pp. 1113 - 1122.

Tzeng, Y-F and Chiu, N-H, 2003. Two-phase parameter design for the optimisation of the electrical-discharge machining process using a taguchi dynamic experiment. The International Journal of Advanced Manufacturing Technology, Vol. 21, No. 12, pp $1005-1014$.

Tzeng, Y-F and Jean, M-D, 2005. Dimensional quality optimization of high-speed CNC milling process with dynamic quality characteristic. Robotics and Computer-Integrated Manufacturing, Vol. 21, pp. 506-517.

Wang, C.H., 2007. Dynamic multi-response optimization using principal component analysis and multiple criteria evaluation of the grey relation model. International Journal of Advanced Manufacturing Technology, Vol. 32, No. 5-6, pp. 617-624.

Wang, C.H., Tong, L.I., 2005. Optimization of dynamic multi-response problems using grey multiple attribute decision making. Quality Engineering, Vol. 17, No. 1, pp. 1-9.

Wang, J., Ma, Y. and Su, G., 2011. Robust parameter design of dynamic multi-response system: a new integrated method. Asian Journal of Quality, Vol. 12, No. 1, pp. 67-79.

Wasserman, G.S., 1996. Parameter design with dynamic characteristics: A regression perspective. Quality and Reliability Engineering International, Vol. 12, No. 2, pp. 113-117.

$\mathrm{Wu}, \mathrm{F}-\mathrm{C}$ and Yeh, C-H., 2005. Robust design of multiple dynamic quality characteristics. International Journal of Advanced Manufacturing Technology, Vol. 25, No. 5-6, pp. 579-588.

Wu, F.C., 2007. Sequential optimization of parameter and tolerance design for multiple dynamic quality characteristics. International Journal of Production Research, Vol. 45, No. 13, pp. 2939-2954.

Wu, C.F.J., 2009. Robust design of nonlinear multiple dynamic quality characteristics. Computers and Industrial Engineering, Vol. 56, No. 4, pp. 1328-1332.

Wu, F-C., 2015. Robust design of mixing static and dynamic multiple quality characteristics. World Journal of Engineering and Technology, Vol. 3, pp. 72-77.

Wu, D.H., Chien, W.T. and Tsai, Y.J., 2005. Applying Taguchi dynamic characteristics to the robust design of a piezoelectric sensor, IEEE Transactions on Ultrasonics, Ferrolectrics and Frequency Control, Vol. 52, No. 3, pp. 480-486.

Yu, W-L, Wu, S-J and Shiah, S-W., 2010. Experimental analysis of dynamic characteristics on the PEM fuel cell stack by using Taguchi approach with neural networks. International Journal of Hydrogen Energy, Vol. 35, pp. 38-47.

Zhang, L-Y, Ma, Y-Z, Zhu, L-Y and Wang J-J., 2014. A modified particle swarm optimization algorithm for dynamic multiresponse optimization based on goal programming approach. 2014 International Conference on Management Science \& Engineering $\left(21^{\text {th }}\right)$, August 17-19, 2014, Helsinki, Finland.

Zhong, X-F and Liu, S-F., 2009. An improved grey incidence analysis for robust designs with nonlinear multi-response dynamic characteristics. Proceedings of 2009 IEEE International Conference on Grey Systems and Intelligent Services, November 10-12, 2009, Nanjing, China. 


\section{Biographical notes}

Dr. Surajit Pal is a Faculty Member in the Statistical Quality Control and Operations Research Unit of the Indian Statistical Institute, Chennai Centre, India. His fields of interest are quality engineering, process optimization, statistical quality control and multiple response optimization.

Dr. Susanta Kumar Gauri is a Faculty Member in the Statistical Quality Control and Operations Research Unit of the Indian Statistical Institute, Kolkata, India. His fields of interest are quality engineering, process optimization, statistical quality control and multiple response optimization.

Received July 2016

Accepted December 2016

Final acceptance in revised form March 2017 